\title{
A Service Modeling Approach with Business-Level Reusability and Extensibility*
}

\author{
Jianwu Wang ${ }^{1,2}$, Jian $\mathrm{Yu}^{1}$, Yanbo Han $^{1}$ \\ ${ }^{1}$ Institute of Computing Technology, Chinese Academy of Sciences, 100080, Beijing, China \\ ${ }^{2}$ Graduate School of Chinese Academy of Sciences, 100039, Beijing, China \\ \{wjw,yujian,yhan\}@software.ict.ac.cn
}

\begin{abstract}
This paper introduces a novel service modeling approach called VINCA_BS, which can be used by business people to extend the functionality of a servicebased software platform from a business perspective. $V I N C A \_B S$ approach introduces the domain analysis approaches into service modeling and uses the domain requirement commonalities to define our VINCA business service models, which are userunderstandable, business-level reusable service abstractions omitting concrete business details. These service models can be instantiated by different business people according to their actual business requirements that consequently add new functionalities to the platform. The approach has been successfully validated in a service-based e-government project.
\end{abstract}

\section{Introduction}

The service-oriented computing paradigm, which is currently highlighted by Web services technologies and standards, provides an effective means of application abstraction, integration and reuse with its loosely-coupled architecture. As an important part of service-oriented system engineering, service-oriented analysis and design have become a research hotspot. $[1,2,3]$ illustrate their service-oriented analysis and design approaches from different perspectives.

In this paper, we introduce our VINCA Business Service Modeling approach (called VINCA_BS) to facilitating service reusability and extensibility at the business level, which is a key part of our VINCA approach [4, 5]. By adopting the concepts and approaches of domain analysis to service-oriented analysis and design, we envisage the business requirement commonalities of a domain, which are the basis of reusable service design. Based on these business requirement commonalities, VINCA business service models are defined, which are userunderstandable, large-granularity service abstractions omitting the concrete business details. Being the service abstractions of concrete services with similar functionalities, the VINCA business service models have high reusability and can be reused by different applications in the same domain. Within the defined domain scope, they can be instantiated and elaborated by different business people to express their own business requirements, bind proper Web services and also guide the implementation of Web services, which consequently can extend the capabilities of serviceoriented software platform at the business level.

The remainder of this paper unfolds in the following manner. Section 2 briefly describes the application scenario of our ongoing e-government project that is the application background of VINCA_BS approach. Section 3 is an overview of the approach. And the key concepts of VINCA_BS approach: VINCA business service model, its modeling approach and its usage guide are respectively detailed in section 4 to 6 . Section 7 discusses related work and our concluding remarks are given in section 8 .

\section{Application background}

Our e-government project is to provide a software platform to facilitate the information sharing between different government departments of Zhaoqing City, Guangdong Province. In the first phase of the project, the platform only supports part of the business applications among eight core government departments. A key requirement of this project is that the platform's functionality needs to be extended by business people

\footnotetext{
"The research work is supported by the National Natural Science Foundation of China under Grant No. 90412005 and 90412010 , the National High-Tech Research and Development Plan of China under Grant No.2003AA414330.
} 
easily, such as adding new departments, adding new business applications. The project investor also wants the platform to be reused in other districts. The business functions provided by different departments are implemented as Web services to facilitate the extension of the platform at the technical level. But it lacks of the reusability and extensibility of services at the business level: How can the business people extend business functions? How can the end-users understand the extended services? For example, what should an employee of the Statistics Bureau do when she needs to add a new business function: "Inquire Monthly Statistics of something particular" to provide some spontaneous statistics information for other related bureaus? What should the functions be depicted as? How can the new added services be understandable to end-users? To solve these problems, we proposed VINCA_BS approach.

\section{Overview of VINCA_BS approach}

VINCA_BS approach is a key part of our proposed VINCA approach that is for end-user-programmable, business-level service composition [4, 5]. VINCA approach includes the key concepts of VINCA business services, service spaces, and Web service virtualization. A VINCA business service is a userunderstandable, large-granularity service abstraction with business-level semantics. Service spaces organize and manage VINCA business services according to the end-users' personalized needs by grouping VINCA business services that either are interrelated or share common features. Web service virtualization is a process of abstracting away the Web service's technical details, describing it with business-related semantics, and then converging semantically substitutive Web services with VINCA business services. After this virtualization process, Web services cannot be seen and used directly, rather they delegate their capabilities to VINCA business services.

Our VINCA_BS approach (shown in Fig.1) follows the convergent engineering methodology, which tries to bridge the gap between business domain and software domain and enable software to adapt to everchanging business [6]. In our approach, VINCA business services are modeled and instantiated from business requirements in a top-down manner; Web services are virtualized in a bottom-up manner; and then VINCA business services and Web services are converged in the middle. For space limitation, this paper mainly discusses the first three steps of the VINCA_BS approach and details of service virtualization and convergence can be found in $[5,7]$.

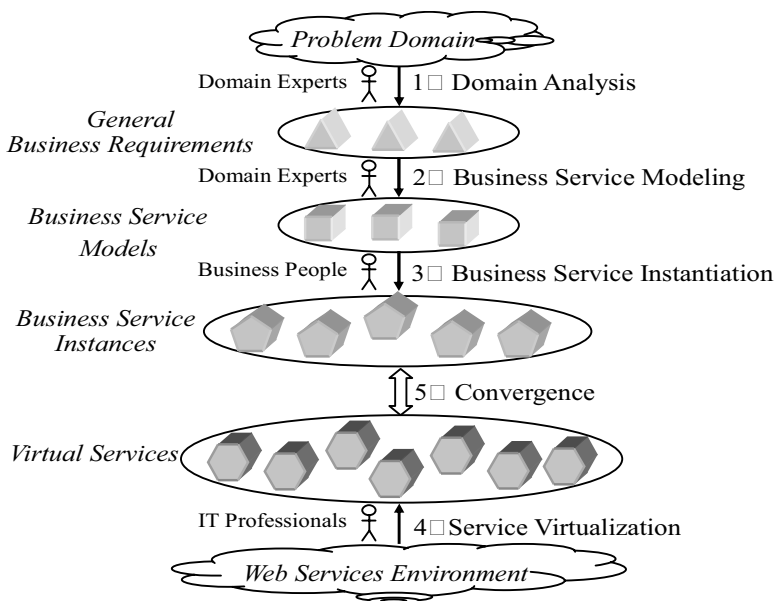

Figure 1. Overview of VINCA_BS approach

\section{VINCA business service model}

A VINCA business service is a kind of businesslevel service that can be directly used by end-users. In this section, we first analyze the characteristics of endusers to indicate the demands on business-level services. The structure of VINCA business service is then illustrated to manifest its usability for end-users. Lastly, the relationship between VINCA business service model and its instances is discussed to show its extensibility.

\subsection{End-user characteristics}

To facilitate the usage of business-level services to end-users, we study and summarize the characteristics of end-users through several real-world projects and list them as follows:

$>$ Business Semantics

End-users usually master their own business, but do not have much IT knowledge. So business-level services depicted by business terms can help them understand the functionalities of services.

$>\quad$ Diversity of Interests in Business Functions

Different end-users have different interests. They may want to know the concrete business details of some business functions that they are interested in, and only the basic information of others. So a kind of description mechanism with different abstraction levels of services can facilitate achieving this goal.

$>\quad$ Personalized Business Requirements

Each end-user has his specific requirements. Even for similar requirements, different end-users may have different demands on functional and non-functional properties. So it will be good if business-level services 
can support the expression of end-users' personalized requirements.

\subsection{Structure of VINCA business service}

VINCA business services are user-understandable, large-granularity service abstractions defined by domain experts according to general business requirements. Their definition is based on domainspecific norms of concepts and functionalities. VINCA business service is annotated with semantics by means of referring to relevant domain ontologies [8]. For instance, VINCA business service ID is formulated based on meaningful combinations of business activities (verbs) and business concepts (nouns) of domain ontologies.

The structure of a VINCA business service includes three aspects (shown in Fig.2): presentation aspect, business configuration aspect and invocation aspect.

\begin{tabular}{|c|}
\hline $\begin{array}{c}\text { Presentation Aspect } \\
\text { (Basic Information) }\end{array}$ \\
\hline $\begin{array}{c}\text { Business Configuration Aspect } \\
\text { (Functional Facets and NFPs) }\end{array}$ \\
\hline $\begin{array}{c}\text { Invocation Aspect } \\
\text { (Inputs and Outputs) }\end{array}$ \\
\hline
\end{tabular}

Figure 2. Structure of VINCA business service model

The division of aspects is founded on the end-users' attention degree in their usage practice: the information of the presentation aspect is interesting to most endusers; the one of the business configuration aspect is interesting to less end-users; the one of the invocation aspect to least end-users. The formal definition of VINCA business service can be found in [5].

1) Presentation Aspect

This aspect depicts the basic information of the VINCA business service, including ID, label, and so on. This aspect contains the information presented to end-users when they log on the service space, which reflects the commonalities of domain business requirements.

2) Business Configuration Aspect

This aspect depicts the concrete business-related details of the VINCA business service, including functional facets [9] (such as the geographical scope of a weather forecast service) and non-functional properties (such as provider and cost of a weather forecast service), which reflect the variabilities of domain business requirements.

3) Invocation Aspect

This aspect depicts the invocation semantics of the VINCA business service, including input parameters and output parameters, which tell end-users how to invoke this service.

All the information of VINCA business services are business related and described by business terms, so they can be understood and used by end-users. Different end-users will know the different information through consulting different aspects of VINCA business services, and express their own requirements by means of specifying the business configuration items. With the support of visual VINCA programming and business service management tools, end-users can find and use needed VINCA business services to fulfill their business goal easily.

\subsection{VINCA business service model and instance}

Domain experts hardly know all the concrete business requirement details, so it is impossible for them to define all VINCA business services. Common business people master their own business but may lack of necessary knowledge of the whole domain. And it is very difficult for them to define their VINCA business services because they may not understand the concepts of VINCA business services and Web services.

So we distinguish two concepts of VINCA business service: VINCA business service model and VINCA business service instance. They are respectively created by domain experts and common business people. Each VINCA business service model can be instantiated to different VINCA business service instances. The definition process is as follows: first, the domain experts utilize common business requirements to define VINCA business service models (step 2 in Fig. 1). Then business people assign value to each item of a certain VINCA business service model's business configuration aspect according to their own business, resulting as corresponding VINCA business service instances (step 3 in Fig. 1), which contain the concrete business details.

Fig. 3 shows an example of a VINCA business service model and its two instances in our egovernment project. The VINCA business service model provides the function of inquiry of related periodical data presented in a form format. The corresponding VINCA business service instances provide the function of monthly statistics data inquiry provided by the statistics bureau and the function of yearly agro-economics data inquiry provided by the agriculture bureau. The IDs of VINCA business service instances are made through respectively substituting 'Monthly-Statistics' and 'Yearly-AgroEconomics' for 'Periodical-data' part of the ID of 
VINCA business service model. (Notes: in this figure, \# denotes it's a fragment of ontology item, FFs denotes functional facets and NFPs denotes non-functional properties).

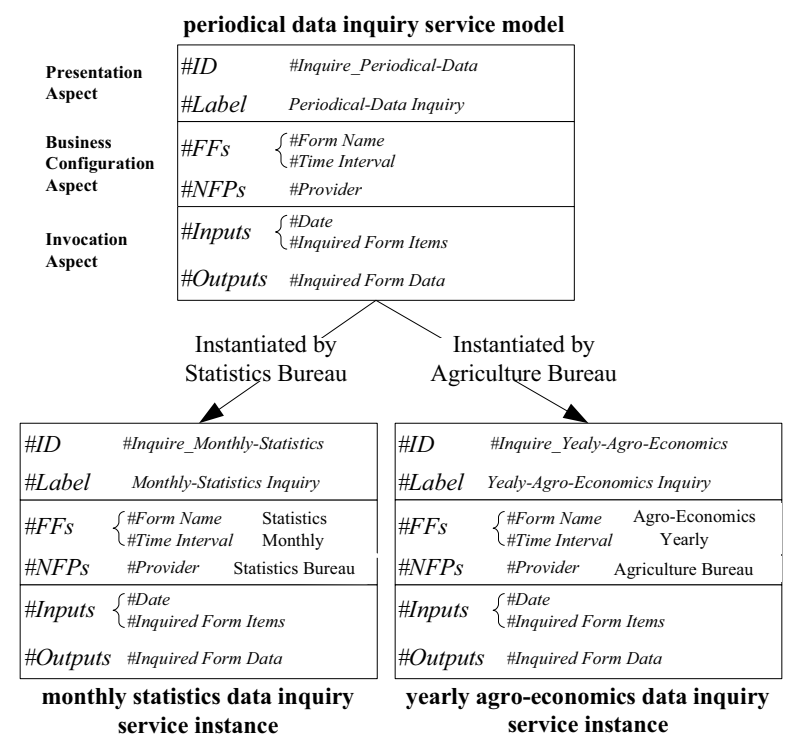

Figure 3. Example of a VINCA business service model and its instances

The relationship between VINCA business service model and VINCA business service instance is similar to the one between class and object. Class is an abstract of similar objects, and the VINCA business service model is also an abstract of similar VINCA business service instances. As VINCA business service models describing the common business requirements, they can be reused easily in other service-oriented software platforms of similar domains. Even after the platform has been built and transferred for use, it can still be extended at the business level through business people instantiating the VINCA business service models.

\section{VINCA_BS approach and its application in e-government project}

As large-granularity computing resources, Web services can be reused easily at the technical level with support of corresponding standards and technologies. Domain analysis is associated with reuse; its purpose is to capture information involved with the domain to be reused in developing further applications of the same domain [10]. The general output of domain analysis is domain model which mainly includes domain scoping, commonality analysis, domain dictionary, requirements engineering [11]. Based on the outcome of domain analysis, highly reusable business-level services can be defined. Now, we will illustrate the detailed steps of our approach through the examples of our e-government project, which is an elaboration of the steps shown in Fig. 1:

1) Domain Boundary Identification

Domain experts identify the domain boundary as the basis of service design. Applications of egovernment are usually classified into three categories: Government to Government (G2G), Government to Business (G2B) and Government to Citizens (G2C). Our project belongs to $\mathrm{G} 2 \mathrm{G}$, so the systems within a certain government department are not included.

2) Domain Requirements Analysis

The domain's business requirements need to be analyzed by domain experts from the business respective, and the concrete business details don't need to be counted in. For our e-government project, the general domain requirements are sharing information among government departments. The information includes data and official document. The manners of information transferring include reporting to the superior, notifying the inferior and inquiring between peer departments.

3) General Business Function Derivation

Basing on the above business requirements, domain experts determine which general business functions are needed to meet the requirements and whether the business functions can be incorporated. To the egovernment project, the processing of data and official document are different for the data may need computation, so the business functions for data and official document need to be designed separately. The three manners of official document transferring requirement are similar from the technical perspective, and can be realized as one business function: 'transfer official document'. The business functions for data processing can be sorted into 'collect data' and 'inquire data' for the data owners of these two ways are different.

4) Business Service Modeling

Each general business function needs to be refined by domain experts from the service perspective to produce VINCA business service models. In this step, each item of VINCA business service models should be specified and related to a proper ontology item. To the e-government project, the time manner of information transferring includes periodical and occasional, which are different at the business configuration aspect and invocation aspect. So each business function can be respectively classified into periodical and occasional category. The periodical data inquiry service model shown in Fig. 3 is an example of VINCA business service model.

5) Business Service Instantiation 
Referring to proper VINCA business service models, business people can assign values to the items of their business configuration aspects according to their own business to get corresponding VINCA business service instances that accomplish certain concrete business functions. Note that this instantiation step can be performed repeatedly by different business people during the whole lifecycle of the platform to realize its incremental and on-demand extension. In our project, business people of different departments will specify VINCA business service models according to their duties. The periodical statistics data inquiry service instance and agro-economics data inquiry service instance shown in Fig. 3 are examples of VINCA business service instances.

6) Web Service Implementation

IT professionals can then program to implement the corresponding Web services according to functional and non-functional requirements depicted in VINCA business service instances.

7) Convergence of Business Service and Web Service

After the implementation of Web services, IT professionals need to register them to VINCA service space to realize the service virtualization and binding to VINCA business service instances. Now the VINCA business service instances are executable.

From the above steps and examples, we demonstrate that how our service modeling approach enable VINCA business service models reusable and extendable at the business level. And the role-based work division is reasonable: the domain experts can identify VINCA business service models without knowing the business details; by referring to the VINCA business service models, business people can easily describe their business details with the prompt of tools, without needing to understand the concepts of VINCA business services and Web services.

\section{Usage of VINCA business service}

After the VINCA business service models are defined by domain experts and further instantiated by business people, they are ready for end-users to use. End-users can understand the VINCA business services since they contain business semantics specified by ontologies. And they can obtain services' detailed information from VINCA business services' three aspects. The VINCA business service models are a sort of abstractions of VINCA business service instances with similar functionalities, so they can be used to manage the instances as a classification to facilitate end-users finding required services.
End-users can use VINCA business services by means of directly selecting VINCA business service instances or configuring on VINCA business service models. For instance, an end-user can either execute the periodical statistics data inquiry service straightly to know the statistics data, or specify the 'form name' item of periodical data inquiry service as 'Statistics Form'. Other contents of service usage, such as the organization of VINCA business services, are elaborated in [5].

\section{Related work}

VINCA business service model and modeling approach are two key concepts of this paper, so we will discuss the related work from two perspectives: service description model and modeling approach.

\subsection{Service description model}

Many researches about service description have been carried out $[12,13,14,15,16]$, but they usually lack of considerations about how end-users can easily use the service description models to meet their personalized requirements. Let's take as an example of the OWL-S [12], which is a typical work about service description model. Because its 'serviceName' item does not have definition rules, it is difficult for endusers to understand the meaning of services. And it lacks of mechanism for end-users to express their functional and non-functional requirements.

\subsection{Service modeling approach}

[1] discusses the approach of service design, which is structured in three phases: from requirements specification to conceptual design and implementation. It is a goal-oriented approach to get needed actions and implement the actions with WSDL and BPEL4WS. [2] discusses how to assess the conformance between the service specification which is from top-down business process decomposition and its design which is from bottom-up service design. These two approaches are mainly concerned about how to meet pre-determined requirements, but lack of the mechanism to adapt to dynamic requirements. In [3], business use cases can be gotten through domain decomposition, which are good candidates for business services. But it doesn't explicitly model business services. So the service design results of these three approaches are all Web services, which are very difficult to be directly understood and used by common business people. And then they can not effectively support the service reuse and extension at the business level. 


\section{Conclusion and future work}

With relevant standards and techniques (such as coarse granularity encapsulation and loose coupling), Web services can support reuse easily at the technical level, and the purpose of domain analysis is to provide reuse through analyzing the commonalities of business domain requirements. So by using the outcome of domain analysis as the basis of service design, highly reusable business-level services can be defined. In VINCA_BS approach, VINCA business service models and VINCA business service instances respectively correspond to the commonalities and variabilities of business requirements. And the work of service modeling and instantiation are respectively assigned to domain experts and common business people. This role-based work division can enable the service instantiation to be performed repeatedly during the whole lifecycle by different business people to realize on-demand extension at the business level. Our approach has been successfully applied in the egovernment project to enable the service-oriented platform to be extended independently by business people of the local governments.

VINCA_BS approach is still underway and quite primitive now. In the future, we plan to theorize the approach and validate it by applying to other applications. Work on the personalized service representation and recommendation is also worthy of further research.

\section{References}

[1] E.Colombo, C.Francalanci, and B.Pernici, "Modeling Cooperation in Virtual Districts: A Methodology for Eservice Design", International Journal of Cooperative Information Systems, Vol. 13, No. 4, 2004, pp.369-411.

[2] D.Quartel, R.Dijkman, and M.v.Sinderen, "Methodological Support for Service-oriented Design with ISDL", Proceedings of the 2nd International Conference on Service-Oriented Computing, New York, November, 2004.

[3] A.Arsanjani, "Service-oriented Modeling and Architecture", http://www-128.ibm.com/developerworks/ webservices/library/ws-soa-design1/, 2004.

[4] Y.Han, H.Geng, et al, "VINCA - A Visual and Personalized Business-level Composition Language for Chaining Web-based Services", Proceedings of the 1st
International Conference on Service-Oriented Computing, LNCS 2910, Springer-Verlag, 2003, pp.165-177.

[5] J.Yu, J.Wang, et al, "Developing End-User Programmable Service-Oriented Applications with VINCA", Proceedings of the 2nd Ljungby Workshop on Information Logistics, Ljungby, Sweden, 2004.

[6] D.Taylor, Business Engineering with Object Technology, John Wiley \& Sons, 1995.

[7] J.Fang, S.Hu and Y.Han, "A Service Virtualization Mechanism for Business End Programming", Chinese Journal of Computers, Vol. 28, No. 4, 2005, pp.549-557 (in Chinese).

[8] N. Guarino, "Formal Ontology in Information Systems," Proceedings of the 1st International Conference on Formal Ontologies in Information Systems, 1998, pp. 3-15.

[9] M.Hafedh, A.Estelle, et al, "Another Nail to the Coffin of Faceted Controlled-Vocabulary Component Classification and Retrieval", Proceedings of the 1997 Symposium on Symposium on Software Reusability, 1997, pp. 89-98.

[10] P.D.Rubén, "Domain Analysis: an Introduction", Software Engineering Notes, Vol. 15, No.2, 1990, pp. 47-54.

[11] M.Harsu, "A Survey on Domain Engineering", Report 31, Institute of Software Systems, Tampere University of Technology, December 2002, available at http://practise.cs.tut.fi/pub/papers/domeng.pdf

[12] The OWL-S Coalition, "OWL-S 1.0 (Beta) Draft Release", http://www.daml.org/services/owl-s/1.0/, 2003.

[13] K.Sivashanmugam, J.Miller, et al, "Framework for Semantic Web Process Composition, Semantic Web Services and Their Role in Enterprise Application Integration and ECommerce", Special Issue of the International Journal of Electronic Commerce, February 2004.

[14] R.Akkiraju, K.Verma, et al, "Executing Abstract Web Process Flows", Proceedings of the 14th International Conference on Automated Planning and Scheduling, British Columbia, Canada, June 2004.

[15] M.Paolucci, T.Kawamura, et al, "Semantic Matching of Web Services Capabilities", Proceedings of 1st International Semantic Web Conference, Sardinia, Italy, June 2002.

[16] P. Oaks, A.t.Hofstede and D. Edmond. "Capabilities: Describing What Services Do", Proceedings of 1 st International Conference on Service-oriented Computing, Trento, Italy, Springer-Verlag, December 2003. 